\title{
Erratum: Spatially Assisted Schwinger Mechanism and Magnetic Catalysis [Phys. Rev. Lett. 117, 081603 (2016)]
}

\author{
Patrick Copinger and Kenji Fukushima \\ (Received 9 February 2017; published 3 March 2017)
}

DOI: 10.1103/PhysRevLett.118.099903

In our previous Letter we presented the analysis for the Sauter-type profile of magnetic fields as a solvable example; $\boldsymbol{B}(x)=B \operatorname{sech}^{2}\left(\kappa x_{1}\right) \boldsymbol{e}_{3}$ [or Eq. (6) in the Letter]. Then, based on the eigenvalue spectrum, we claimed that the mass squared should be shifted as $m^{2} \rightarrow m^{2}-\kappa^{2}$. However, there was a sign mistake in Eq. (11) in our Letter and the correct one (that is consistent with Ref. [1]) is

$$
\lambda_{n}^{ \pm}=p_{2}^{2}\left[1-\frac{(e B)^{2}}{\left(\kappa^{2} \tilde{n}-\left|\kappa^{2} / 2 \pm e B\right|\right)^{2}}\right] \mp e B-\left(\kappa^{2} \tilde{n}^{2}-2 \tilde{n}\left|\frac{\kappa^{2}}{2} \pm e B\right|+\frac{\kappa^{2}}{4}\right),
$$

where a half integer $\tilde{n} \equiv n+1 / 2$ ranges with $n \in\left[0,\left|\frac{1}{2} \pm\left(e B / \kappa^{2}\right)\right|-\frac{1}{2}-\sqrt{p_{2} e B / \kappa^{3}}\right)$ for $\lambda_{n}^{ \pm}$. We can significantly simplify the above expression for $e B>\kappa^{2} / 2$, which is the case for our interested situation with small inhomogeneity. Then, Eq. (12) in the Letter must be replaced with

$$
\lambda_{n}^{ \pm}=\left(1-\frac{p_{2}^{2} \kappa^{2}}{\left(e B-\kappa^{2} n^{ \pm}\right)^{2}}\right) n^{ \pm}\left(2 e B-\kappa^{2} n^{ \pm}\right)
$$

where $n^{+}=n$ and $n^{-}=n+1$. Contrary to our Letter, the correct lowest eigenvalue is $\lambda_{0}^{+}=0$, not negative.

Let us explain why the above changes would not affect our claim of our Letter. For this purpose we first point out an interesting property of Eq. (2). Previously we assumed that the smallest eigenvalue was found for $p_{2}=0$, but we note that the coefficient of the $p_{2}^{2}$ term is negative. This means that $\lambda_{n}^{ \pm}$is smaller for larger $p_{2}^{2}$. The range of $p_{2}$ is bounded by the condition for the existence of $n \geq 0$. Therefore, the next smallest eigenvalue in both branches of $\lambda_{n}^{ \pm}$is $\left.\lambda_{1}^{+}\right|_{p_{2}=\left(e B-\kappa^{2}\right) /(\kappa e B)}=$ $\left.\lambda_{0}^{-}\right|_{p_{2}=\left(e B-\kappa^{2}\right) /(\kappa e B)} \simeq 4 \kappa^{2}$ for small $\kappa^{2}$. Thus, the energy gap is open not by the scale of $e B$ but the scale of $\kappa^{2}$.

Then, one may think of a possibility to analytically continue the theory as $\kappa^{2} \rightarrow-\kappa^{2}<0$ to realize $m^{2} \rightarrow m^{2}-4 \kappa^{2}$, but such a modification on the Sauter-type potential is not straightforward because the treatment of the boundary condition should be changed accordingly. So, we now employ a diagrammatic approach for systematic evaluation, which also gives an explanation for another misleading point, namely, Eq. (17) in our Letter.

Previously, we gave an independent argument for $m^{2} \rightarrow m^{2}-\kappa^{2}$ from the Gaussian approximation. The vector potential corresponding to the magnetic field in Eq. (14) in our Letter, i.e., $\boldsymbol{B}(x)=\left[B-\frac{1}{2} B \kappa^{2}\left(x_{1}^{2}+x_{2}^{2}\right)\right] \boldsymbol{e}_{3}$ should be

$$
\boldsymbol{A}(x)=\frac{B}{2}\left(x_{1} \boldsymbol{e}_{2}-x_{2} \boldsymbol{e}_{1}\right)-\frac{B \kappa^{2}}{6}\left(x_{1}^{3} \boldsymbol{e}_{2}-x_{2}^{3} \boldsymbol{e}_{1}\right) .
$$

Our previous analysis neglected the quantity in the second parentheses since they are non-Gaussian fluctuations. The correct expansion in terms of $\kappa^{2}$ should reproduce the exact answer for the Sauter-type profile, that is, zero. We can clearly confirm this expectation by computing the ground state energy using the Lagrangian,

$$
L=\frac{\dot{x}_{1}^{2}+\dot{x}_{2}^{2}}{4}+\frac{i e B}{2}\left(-x_{2} \dot{x}_{1}+x_{1} \dot{x}_{2}\right) \pm e B \mp e B \kappa^{2}\left(x_{1}^{2}+x_{2}^{2}\right)+\frac{i e B \kappa^{2}}{3}\left(x_{2}^{3} \dot{x}_{1}-x_{1}^{3} \dot{x}_{2}\right)
$$

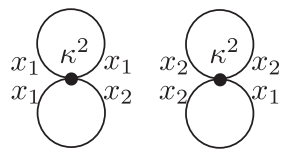

FIG. 1. One-loop and two-loop diagrams. 
For the unperturbed part the ground state energy is zero due to the Landau zero mode. The Gaussian approximation corresponds to the one-loop diagrams in Fig. 1 with a vertex, $\mp e B \kappa^{2}\left(x_{1}^{2}+x_{2}^{2}\right)$. Simple calculation leads to $\pm \frac{1}{2} \kappa^{2}$, and this $-\kappa^{2}$ explains $e^{\kappa^{2} T}$ in Eq. (17) in our Letter. We find that the two-loop diagrams in Fig. 1 give $+\frac{1}{2} \kappa^{2}$. Then, the complete result at the $\kappa^{2}$ order is $\pm \frac{1}{2} \kappa^{2}+\frac{1}{2} \kappa^{2}$, and the lowest is zero. This perturbative approach allows us to flip the sign of $\kappa^{2}$. The one loop is not changed but the sign of the two loop only is changed. So, the ground state energy is then $\pm \frac{1}{2} \kappa^{2}-\frac{1}{2} \kappa^{2}$, and the lowest is $-\kappa^{2}$. This negative energy in the ground state leads to a mass shift by $m^{2} \rightarrow m^{2}-\kappa^{2}$, as anticipated in our Letter.

To summarize, the conclusion of our Letter is unchanged and the enhancement results from inhomogeneous magnetic fields, with a correction of the magnetic profile from decreasing (negative curvature) behavior to increasing (positive curvature) behavior.

[1] D. Cangemi, E. D’Hoker, and G. V. Dunne, Phys. Rev. D 52, R3163 (1995). 